\title{
BIM 4D NO PLANEJAMENTO DE OBRAS: DETALHAMENTO, BENEFÍCIOS E DIFICULDADES
}

\section{BIM 4D IN CONSTRUCTION SCHEDULING: DETAILS, BENEFITS AND DIFFICULTIES}

\author{
Paula Heloisa da Silva ${ }^{1}$ \\ Universidade Federal do Paraná, Curitiba, PR, Brasil, paulaheloisa.s@hotmail.com \\ Julianna Crippa ${ }^{2}$ \\ Universidade Federal do Paraná, Curitiba, PR, Brasil, julianna.crippa@gmail.com \\ Sergio Scheer ${ }^{3}$ \\ Universidade Federal do Paraná, Curitiba, PR, Brasil, sergioscheer@gmail.com
}

\begin{abstract}
Resumo
Os processos de Planejamento e Controle de Obras (PCO) subsidiam o cumprimento do custo, prazo e qualidade esperado da obra. Estes apresentam influência direta sob a produtividade obtida no canteiro de obras, pois as informações falhas provenientes da documentação gerada são interpretadas como os principais fatores de causa-efeito associados à baixa produtividade, desperdícios de materiais e mão de obra, resultando em baixa qualidade do produto final. O uso da Modelagem da Informação da Construção na construção civil vem sendo proposto como uma solução tecnológica para tais problemas. Quando BIM é associado ao PCO é recorrente utilizar o termo BIM 4D ou modelagem 4D. Desta forma, este artigo busca esclarecer como desenvolver o uso do BIM 4D para PCO e quais os benefícios e dificuldades provenientes. A questão foi abordada por meio de uma Revisão Sistemática da Literatura. Identificou-se o sequenciamento de atividades para o desenvolvimento do PCO baseado em BIM e ferramental associado. As dificuldades principais encontradas foram associadas ao processo de implementação e ao processo trabalhoso e intenso da modelagem 3D requerida e da integração disciplinada com o cronograma da obra. Entretanto, o BIM 4D para o PCO mostra-se viável apontando benefícios no provimento de diretrizes de otimização do processo tradicional de PCO, trazendo soluções para grande parte dos problemas inerentes ao método convencional, além de contribuir para a redução de retrabalhos por meio da interoperabilidade, integração de sistemas de comunicação e simulação do processo construtivo.
\end{abstract}

Palavras-chave: BIM 4D. Planejamento. Construção. Processo.

\begin{abstract}
The Plan of Construction Operations (PCO) subsidizes the fulfillment of the cost, term and the expected quality of the work. These have a direct influence on the productivity obtained at the construction site, since information flaw from the generated documentation is interpreted as the main cause-and-effect factor associated with low productivity, waste of materials and workmanship, resulting in poor quality of the final product. The use of Building Information Modeling in civil construction has been proposed as a technological solution for such problems. When BIM is associated with PCO it is recurrent to use the term 4D BIM or 4D modeling. In this way, this article seeks to clarify how to develop the use of 4D BIM for PCO and what benefits and difficulties arise. The issue was addressed through a Systematic Literature Review. Activity sequencing was identified for the development of BIM-based $P C O$ and associated tooling. The main difficulties encountered were associated with the implementation process and the laborious and intense process of 3D modeling required and the disciplined integration with the work schedule. However, 4D BIM for PCO is feasible, showing benefits in providing optimization guidelines for the traditional PCO process, providing solutions for most of the problems inherent in the conventional method, as well as contributing to the reduction of rework through interoperability, integration of communication systems and simulation of the construction process.
\end{abstract}

Keywords: BIM 4D. Planning. Construction. Process.

How to cite this article:

SILVA, Paula Heloisa da; CRIPPA, Julianna; SCHEER, Sergio. BIM 4D no planejamento de obras: detalhamento, benefícios e dificuldades. PARC Pesquisa em Arquitetura e Construção, Campinas, SP, v. 10, p. e019010, fev. 2019. ISSN 1980-6809. Disponível em: <https://periodicos.sbu.unicamp.br/ojs/index.php/parc/article/view/8650258>. Acesso em: 26 fev. 2019. doi:https://doi.org/10.20396/parc.v10i0.8650258. 


\section{Introdução}

Os projetos de edificações são assumidos como projetos de alto índice de complexidade, devido a quantia de dados que estes produzem em formatos de desenhos, grafos, planilhas e documentos (MATTHEWS et al., 2015; SHEN et al., 2010). Para tanto, o sucesso desses projetos complexos, está diretamente associado à qualidade da informação que esses possuem (SAINI; MHASKE, 2013).

$\mathrm{Na}$ indústria da construção civil, a tomada de decisão é apoiada em informações inconsistentes e sem o nível de detalhe necessário (BIOTTO; FORMOSO; ISATTO, 2012). Este cenário representa um dos maiores problemas contemporâneos da indústria. Segundo Matthews et al., (2015) ter acesso à informação correta, no local correto e no momento necessário condiciona o encaminhamento do projeto e é um fator fundamental para a obtenção de qualidade.

Os processos de Planejamento e Controle de Obras (PCO) são processos complementares que subsidiam o cumprimento do custo, prazo e qualidade esperado da obra. Estes apresentam influência direta sob a produtividade obtida no canteiro de obras, pois as informações provenientes destes documentos são interpretadas como os principais fatores de causa-efeito associados à baixa produtividade, desperdícios de materiais e mão de obra e baixa qualidade do produto final (BRITO; FERREIRA, 2015).

Os autores Ahankoob et al. (2012), Brito e Ferreira (2015) pontuam que o método convencional de produzir os cronogramas é baseado no Método do Caminho Crítico (Critical Path Method - CPM) e Cadeia Crítica (Critical Chain). Ambos são fundamentados pela associação e encadeamento das tarefas de construção, com o tempo despendido para a realização destas e a alocação de recursos necessários.

Por mais que existam ferramentas para apoiar o método tradicional de produzir cronogramas de obra, este ainda depende de muitos processos manuais necessitando de uma quantia significativa de tempo para a retroalimentação de informações conforme ocorrem as revisões de projeto, resultando em informações de baixa qualidades e consequentemente planejamentos inconsistentes, aumentos excessivos de custos e prazos (LI et al., 2009; MIKULAKOVA et al., 2010; FENG; CHEN; HUANG, 2010; AHANKOOB et al., 2012; PITAKE; PATIL, 2013; LIU; AL-HUSSEIN; LU, 2015).

Outra dificuldade enfrentada pela indústria da construção civil é a visualização e interpretação do cronograma proveniente do método tradicional. Por se tratar de um documento unidimensional, é abstrato para os trabalhadores (AHANKOOB et al., 2012; BRITO; FERREIRA, 2015).

Ainda, a fragmentação de processos e fluxos informacionais também é um problema presente neste setor, contribuindo para documentação de projetos incompatíveis e tomada de decisão equivoca por falta de integração na comunicação (BRITO; FERREIRA, 2015).

Quanto ao controle de obra, Tserng, Ho e Jan (2014) afirmam que a atualização periódica do cronograma de obra, é essencial para a eficiência do monitoramento e controle das etapas de construção. Usualmente este processo é realizado de forma manual, sendo uma tarefa morosa, tendendo a prover informações inconsistentes, resultando em atrasos e aumento de custos.

Conforme apontado pelos autores Saini e Mhaske (2013) e Luke et al. (2014) existe uma carência de investimentos tecnológicos do setor construtivo, para otimizar a qualidade das informações que alimentam projetos e processos de PCO. O uso da Modelagem da Informação da Construção (Building Information Modeling - BIM) na construção civil vem 
sendo proposto como uma solução tecnológica para tais problemas. Quando BIM é associado ao PCO é recorrente utilizar o termo BIM 4D ou modelagem 4D (CIC, 2011; EASTMAN et al., 2011, p.24; VA, 2010; CBIC, 2016).

Em que pese, os estudos acerca de BIM tiveram início na década de 70 , sendo inicialmente citados por Charles M. Eastman (QUIRK, 2012). BIM é uma metodologia de projeto que subsidia a integração da informação ao longo das etapas de processo de projeto, gestão de projetos e obras (BRITO; FERREIRA, 2015). Devido a essas características, o BIM permite que os envolvidos no projeto possam ter acesso às informações consistentes ao longo das etapas do projeto, contribuindo para a precisão dos documentos e tomada de decisão (AHANKOOB et al. 2012). Neste cenário é valido pontuar que as pesquisas inerentes à interoperabilidade tiveram maior incidência a partir dos anos 2000, por meio do trabalho dos autores Laiserin (2002) e Eastman (2002).

Ao tratar-se de $\mathrm{PCO}$ é necessário adentrar às discussões acerca da quarta dimensão de BIM. A modelagem BIM 4D é definida como a integração de modelos 3D com a dimensão temporal (WANG et al., 2014). Esta integração resulta em cronogramas mais assertivos e maior controle de prazos, segundo Brito e Ferreira (2015). Tais melhorias são possíveis devido à conexão das dimensões espaciais com a temporal promovidas pela visualização em modelos 3D e simulação de sequência de atividades da obra, que induzem a confiabilidade dos cronogramas e a melhoria da gestão da comunicação.

O presente artigo tem como objetivo analisar a viabilidade do uso de BIM 4D para PCO, identificando a forma de realiza-lo e as potencialidades e dificuldades inerentes a este uso por meio de uma Revisão Sistemática da Literatura (RSL).

\section{Metodologia}

De acordo com Kitchenham et al. (2009) este método permite identificar, avaliar criticamente, interpretar e consolidar as pesquisas disponíveis de modo a fornecer os subsídios necessários para responder a dúvida de uma pesquisa específica ou área de interesse.

A RSL é um estudo de caráter secundário que são utilizados para agregar resultados de estudos primários acerca da proposta de pesquisa previamente estabelecida (DRESCH; LACERDA; ANTUNES JÚNIOR, 2015). A presente pesquisa foi desenvolvida de acordo com a metodologia de RSL de Brereton et al. (2007). Segundo os mesmos autores, realizar uma revisão sistemática da literatura envolve várias atividades discretas, que podem ser agrupadas em três fases principais: planejamento, condução e documentação. $O$ processo geral da revisão é sistematizado em 10 estágios, conforme é apresentado na Figura 1.

Figura1 - Processo de Revisão Sistemática da Literatura

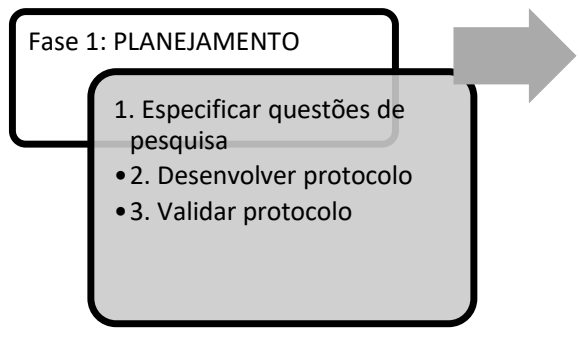

Fonte: Adaptado de Brereton et al. (2007).
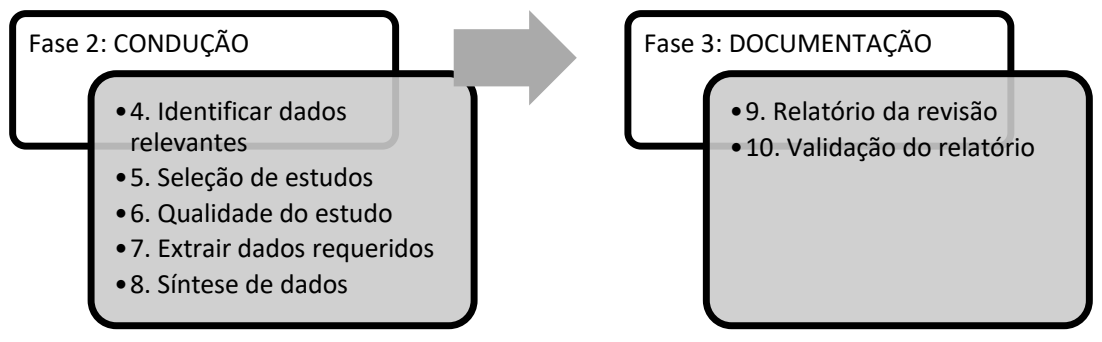
De acordo com Biolchini et al. (2007), a RSL possibilita a sintetização do conhecimento proveniente de uma série de dados, este que por sua vez é resultado de um processo de coleta rígido. Este processo deve ser rigorosamente estruturado de modo que os dados obtidos sejam apenas aqueles de fato relevantes para a pesquisa.

Como questão de pesquisa foi estabelecido identificar: Como se desenvolve o uso da Modelagem da Informação da Construção com o BIM 4D para o processo de planejamento de obras e quais os benefícios e dificuldades provenientes?

O protocolo da busca foi definido pelos termos de busca e locais de busca. Determinouse as bases de dados: Science Direct, Scopus e CAPES, complementada pelo mecanismo de busca Google Scholar. Os termos estabelecidos foram: BIM 4D, BIM 4D construction planning, BIM 4D construction planning process, BIM 4D construction planning process, feasibility BIM 4D construction planning process e construction scheduling \& BIM. A busca foi restrita a localização dos termos no título, resumo e palavras-chave das publicações. As buscas ocorreram no período de 2010 a 2017.

Na fase de condução da RSL, visando identificar os trabalhos com relevância para subsidio a síntese da questão estabelecida, houve a necessidade definir os critérios de inclusão e exclusão de estudos (DRESCH; LACERDA; ANTUNES JÚNIOR, 2015). Desta maneira, definiu-se excluir da amostra de estudo conteúdo como monografias, dissertações e teses, da mesma forma que guias, tutoriais, manuais ou livros, enfatizando assim apenas em artigos publicados em eventos ou periódicos por apresentarem conhecimento revisado por pares.

Também foram eliminadas as publicações repetidas assim como as que não tinham foco principal do estudo o PCO e BIM. Os documentos, nesta triagem, tiveram seus resumos lidos e avaliados, excluíndo-se aqueles não que relacionavam modelos BIM 3D com o planejamento de obras, além de artigos escritos em outras línguas que não haviam sido identificados para a exclusão prévia.

No quesito de conteúdo, também foram excluídos artigos que abordavam somente aspectos muito específicos como: gestão da qualidade, gestão de riscos ou segurança. Assim, foram selecionados somente aqueles que proviam informações acerca dos processos de modelagem BIM 4D, elucidando as etapas necessárias desde a modelagem BIM, o link entre a base de dados e o modelo 3D, até a simulação 4D e os resultados provenientes do uso dessa tecnologia. Também foram considerados relevantes para este trabalho, estudos que utilizaram modelos do tipo CAD 3D ou BIM-based.

Após concluída esta triagem restaram 32 trabalhos selecionados no período de 2010 a 2017, os quais foram lidos na íntegra. Por fim a terceira fase destinou-se a apresentação dos dados coletados a fim da validação da pesquisa.

\section{Caracterização da amostra resultante}

O Quadro 1 registra o desenvolvimento da condução da RSL explicitando a quantidade de documentos encontrados por base de referência por termo de busca entre triagens.

Após realizada a leitura dos trabalhos selecionados para a condução desta RSL, foi possível identificar que o assunto desta pesquisa - detalhamento do uso do BIM para PCO, benefícios e dificuldades - é majoritariamente explorado na Ásia, na América do Norte e Sul respectivamente, tendo menor incidência de investigação na Europa e Oceania. Foi possível verificar também, que dos trabalhos selecionados, nenhum é de origem Africana (Figura 3) 
SILVA, Paula Heloisa da; CRIPPA, Julianna; SCHEER, Sergio.

BIM 4D no planejamento de obras: detalhamento, benefícios e dificuldades

Quadro 1- Detalhamento da condução da RSL
\begin{tabular}{|l|c|c|c|c|c|}
\hline \multicolumn{1}{|c|}{ Termo de busca } & $\begin{array}{c}\text { Google } \\
\text { Scholar }\end{array}$ & $\begin{array}{c}\text { Science } \\
\text { Direct }\end{array}$ & Scopus & Capes & Total \\
\hline BIM 4D & 18900 & 1251 & 602 & 195 & 20948 \\
\hline BIM 4D construction planning & 10600 & 276 & 344 & 38 & 11258 \\
\hline BIM 4D construction planning process & 9910 & 269 & 71 & - & 10250 \\
\hline BIM 4D \& construction planning process & 194 & - & - & - & 194 \\
\hline Feasibility BIM 4D construction planning process & - & 108 & - & - & 108 \\
\hline Construction Scheduling \& BIM & 725 & 52 & - & 10 & 787 \\
\hline \multicolumn{1}{|c|}{ Triagem } & $\begin{array}{c}\text { Google } \\
\text { Scholar }\end{array}$ & $\begin{array}{c}\text { Science } \\
\text { Direct }\end{array}$ & Scopus & Capes & Total \\
\hline Análise dos títulos e eliminação dos repetidos & 17 & 19 & 12 & 7 & 52 \\
\hline $\begin{array}{l}\text { Eliminação de artigos fora do contexto (não } \\
\text { abordam BIM) }\end{array}$ & 13 & 11 & 5 & 3 & 32 \\
\hline
\end{tabular}

Fonte: Os autores.

Figura 3: Análise da distribuição global de percentual de quantidade das pesquisas aderidas

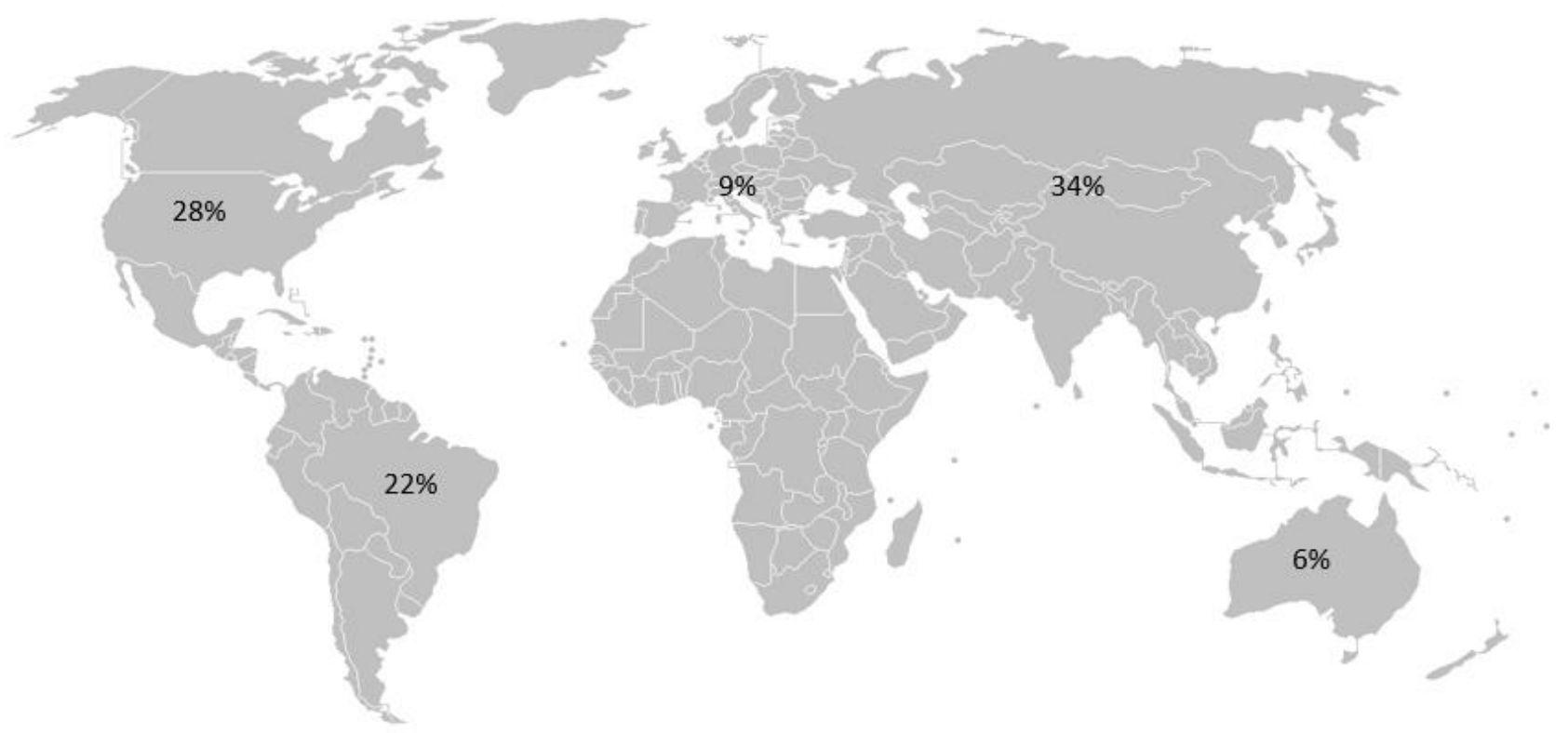

Fonte: Os autores.

A análise dos veículos de publicação destacou o periódico internacional Automation in Construction com $34 \%$ da quantia total dos estudos desta amostra e apontou o periódico nacional Ambiente Construído e o evento Encontro Nacional de Tecnologia no Ambiente Construído, com um acumulado de 12\% dos estudos desta amostra (Tabela 1).

\section{Resultados e discussão}

De modo geral, o processo de PCO com BIM foi aplicado igualmente em todos os artigos lidos, apresentando variações apenas quanto às ferramentas utilizadas em cada etapa. O desenvolvimento desta integração é composta por uma série de processos regrados pela metodologia BIM. Deste modo, Silva, Scheer e Arruda (2017) sintetizaram um esquema gráfico (Figura 4) que ilustra os fluxos para a elaboração do cronograma e posteriormente o controle da obra integrado com BIM. A necessidade de otimização da qualidade da informação para subsidiar os processos de PCO é intrínseca. Apesar da conscientização dos engenheiros e arquitetos quanto a importância do controle diário de cronograma, esta atividade por vezes não é realizada apropriadamente, pois está diretamente associada com grande consumo de tempo, tendendo a apresentar inconsistência nas informações por ser realizada manualmente (KIM et al., 2013). O 
SILVA, Paula Heloisa da; CRIPPA, Julianna; SCHEER, Sergio.

BIM 4D no planejamento de obras: detalhamento, benefícios e dificuldades

presente contexto suscita a carência de redução de processos manuais realizados para o PCO, assim como também o seu modo de representação, acesso e distribuição de informações (SAINI; MHASKE, 2013; LUKE et al., 2014).

Tabela 1 - Revista e Anais de congressos selecionados

\begin{tabular}{|c|c|c|c|}
\hline Revistas e eventos & $\begin{array}{l}\text { Tipo: Periódico ou } \\
\text { Evento }\end{array}$ & $\begin{array}{c}\text { Trabalhos } \\
\text { Selecionados }\end{array}$ & $\%$ \\
\hline Automation in Construction & Periódico & 11 & $34 \%$ \\
\hline Advanced Engineering Informatics & Periódico & 2 & $6 \%$ \\
\hline Encontro Nacional da Tecnologia do Ambiente Construído & Evento & 2 & $6 \%$ \\
\hline Ambiente Construído & Periódico & 2 & $6 \%$ \\
\hline Procedia Engineering & Periódico & 2 & $6 \%$ \\
\hline $\begin{array}{c}\text { Annual Workshop of the European Group for Intelligent Computing in } \\
\text { Engineering }\end{array}$ & Evento & 1 & $3 \%$ \\
\hline Computing in Civil Engineering & Periódico & 1 & $3 \%$ \\
\hline Congresso Brasileiro De Engenharia De Produção & Evento & 1 & $3 \%$ \\
\hline Construction Research Congress & Evento & 1 & $3 \%$ \\
\hline $\begin{array}{l}\text { ICCREM: Smart Construction and Management in the Context of New } \\
\text { Technology }\end{array}$ & Evento & 1 & $3 \%$ \\
\hline International Journal of Engineering Trends and Technology (IJETT) & Periódico & 1 & $3 \%$ \\
\hline International Journal of Scientific Engineering and Research (IJSER) & Periódico & 1 & $3 \%$ \\
\hline International Conference on Computing in Civil and Building Engineering & Evento & 1 & $3 \%$ \\
\hline Jordan Journal of Civil Engineering & Periódico & 1 & $3 \%$ \\
\hline Journal of Civil Engineering and Management & Periódico & 1 & $3 \%$ \\
\hline Management in Construction Research Association & Periódico & 1 & $3 \%$ \\
\hline Simpósio Brasileiro de Qualidade do Projeto no Ambiente Construído & Evento & 1 & $3 \%$ \\
\hline Simulation Conference (WSC), Proceedings of the 2012 Winter & Evento & 1 & $3 \%$ \\
\hline
\end{tabular}

Fonte: Os autores.

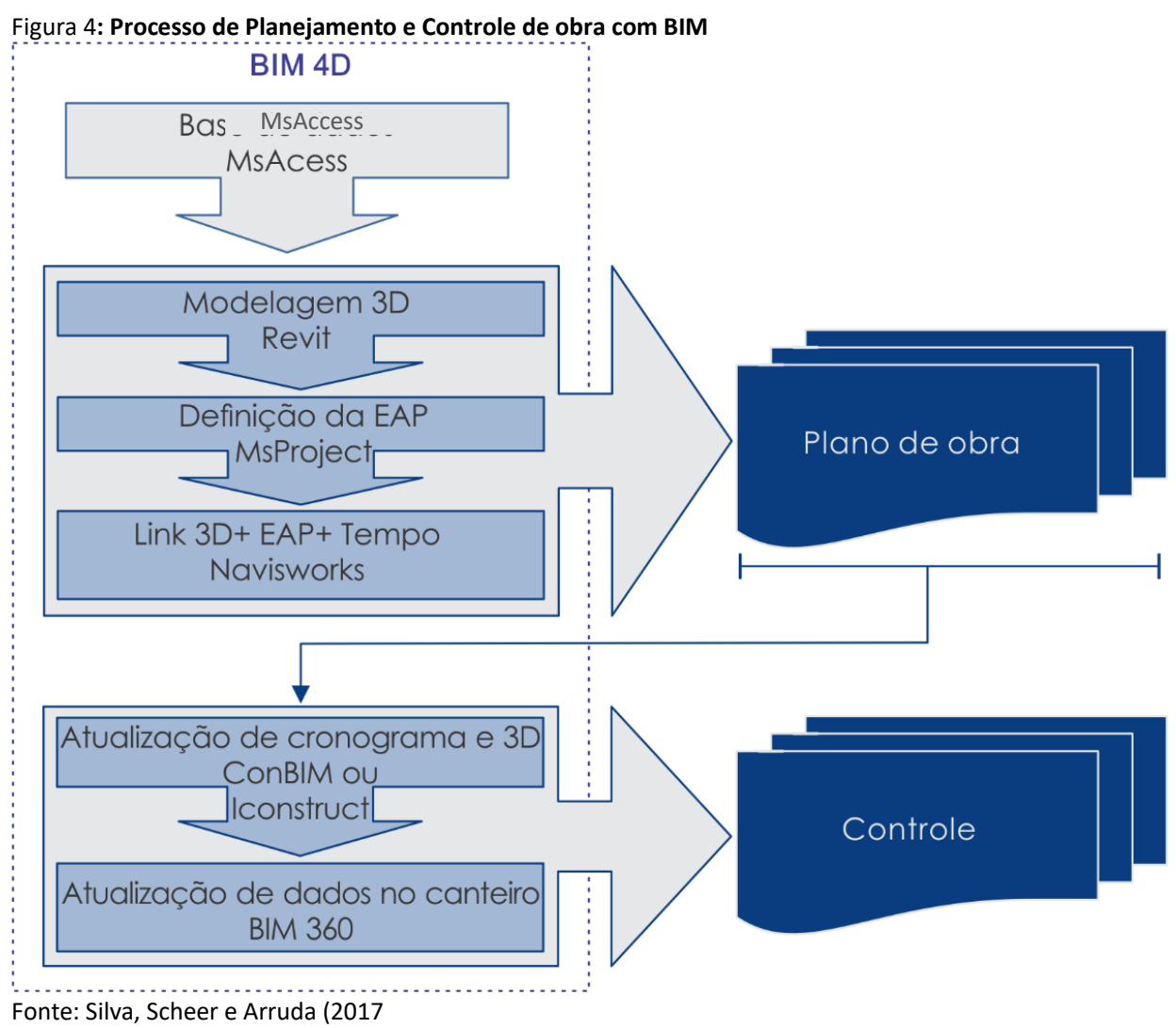


A leitura dos artigos selecionados permitiu evidenciar quais os softwares de uso recorrente, para a viabilização do PCO por meio dos processos BIM, como mostra o Quadro 2. Verifica-se que os softwares mais utilizados para o a obtenção de modelos 4D são: MsAccess para o desenvolvimento de uma base de dados de construção; o Revit para modelagem em 3D; MsProject para a definição dos pacotes de atividades e Estrutura Analítica de Projeto (EAP); para a realização de simulações e integração do cronograma com o modelo 3D, utiliza-se o Navisworks; por fim para realizar o controle de obra usa-se o BIM 360. Também cabe ressaltar que segundo Liu et al. (2014) mesmo existindo diversas ferramentas para simulação BIM 4D que possibilitam excelentes resultados, como os provenientes do Autodesk Navisworks, a maioria dos demais softwares se destinam a apenas à visualização do modelo. Para tanto é necessário incorporar o cronograma de atividades de construção ao modelo 3D, sendo preciso que este seja desenvolvido em um software compatível com a plataforma BIM, como MS Project ou Primavera.

\begin{tabular}{|c|c|c|}
\hline Atividade & Software & $\%$ uso \\
\hline \multirow[t]{5}{*}{ MODELAGEM 3D } & REVIT & $50 \%$ \\
\hline & ARCHICAD & $17 \%$ \\
\hline & MD CAD & $11 \%$ \\
\hline & AUTOCAD 3D & $11 \%$ \\
\hline & VPA (virtual prototype analysis) & $6 \%$ \\
\hline \multirow[t]{7}{*}{ SIMULAÇÕES E INTEGRAÇÃO } & NAVISWORKS & $62.50 \%$ \\
\hline & SIMPHONY & $6.25 \%$ \\
\hline & VISPMIS & $6.25 \%$ \\
\hline & 4D-GCPSU & $6.25 \%$ \\
\hline & STROBOSCOPE & $6.25 \%$ \\
\hline & SITESIM-EDITOR & $6.25 \%$ \\
\hline & SIMULATION TOOLKIT SHIPBUILDING & $6.25 \%$ \\
\hline \multirow[t]{4}{*}{ PLANEJAMENTO } & MSPROJECT & $60 \%$ \\
\hline & EXCEL & $25 \%$ \\
\hline & ALGORÍTMOS GENÉTICOS & $10 \%$ \\
\hline & ASTA POWER PROJECT & $5 \%$ \\
\hline \multirow[t]{5}{*}{ CONTROLE } & BIM 360 & $20 \%$ \\
\hline & ICONSTRUCT & $20 \%$ \\
\hline & CONBIM & $20 \%$ \\
\hline & 4D-GCPSU & $20 \%$ \\
\hline & SIEMENS PLM & $20 \%$ \\
\hline
\end{tabular}

Fonte: Os autores

Já para o processo de controle, os autores Brito e Ferreira (2015) propõem o uso de cores no modelo 4D para representar as atualizações referentes aos processos construtivos em andamento, permitindo identificar desvios entre as atividades planejadas e as executadas. Matthews et al. (2015) propõem o uso do Iconstruct e do BIM 360, tais softwares possibilitam que as informações coletadas no canteiro sejam disseminadas a todos os envolvidos e atualizadas no modelo 4D quase inteiramente de forma automatizada. Os autores Tserng, Ho e Jan (2014) defendem o uso do ConBIM para aumentar a eficiência do controle de obras, este sistema traz melhorias para a atualização do cronograma as-built. Hu e Zhang (2010) propõem o uso do sistema 4D GCPSU. 
Para os autores Saini e Mhaske (2013) e Luke et al. (2014), a tecnologia BIM 4D pode resolver a maioria dos problemas provenientes do método de $\mathrm{PCO}$ tradicional, pois integra o modelo 3D da construção com o seu cronograma em uma única plataforma. Igualmente, Tserng, Ho e Jan (2014), Brito e Ferreira (2015) e Matthews et al. (2015) apontam o uso de modelos BIM 4D como um meio de otimizar o processo de controle e monitoramento de obras, devido a possibilidade de realizar o acompanhamento do progresso da obra em um modelo virtual. De acordo com Hartmann et al. (2012) e Shen et al. (2010), a tecnologia BIM representa digitalmente o progresso construtivo, possibilitando trocas de informações mais consistentes por meio da interoperabilidade do modelo. Saini e Mhaske (2013) afirmam que a modelagem BIM fornece informações com o nível de detalhe necessário para cada envolvido, o que torna o processo de monitoramento mais eficaz

Ao longo das leituras foi possível identificar e categorizar as dificuldades (Quadro 3) e os benefícios (Quadro 4) inerentes aos modelos 4D, permitindo mensurar a incidência destas na RSL. Para tanto, as dificuldades são compreendidas como problemas enfrentados pelos envolvidos no projeto ao usar a tecnologia BIM e os benefícios são vantagens vivenciadas pelos usuários da tecnologia. Em ambos os Quadros 3 e 4, apresenta-se a categoria da dificuldade ou benefício, a percentagem de recorrência de identificação destas nos estudos da amostra e a referências correspondentes.

\begin{tabular}{|c|c|c|}
\hline Recorrência & DIFICULDADES/RESTRIÇÕES & REFERÊNCIAS \\
\hline $30 \%$ & Dificuldade em implementação da tecnologia & $\begin{array}{l}\text { (TSERNG; HO; JAN, 2014; AHANKOOB et al., 2012; } \\
\text { MENDES et al.,2014; MATTHEWS et al.,2015; PITAKE; } \\
\text { PATIL, 2013; HU; ZHANG, 2010; WANG; CHIEN, 2014; } \\
\text { ROLFSEN; MERSCHBROCK, 2016) }\end{array}$ \\
\hline $19 \%$ & $\begin{array}{l}\text { Grande consumo de trabalho intensivo para } \\
\text { implementação efetiva da tecnologia e atualização do } \\
\text { modelo e associação com o cronograma. }\end{array}$ & $\begin{array}{l}\text { (PITAKE; PATIL, 2013; LI et al., 2009; WANG; CHIEN, } \\
\text { 2014; CHEN et al., 2013; TSERNG; HO; JAN, 2014); }\end{array}$ \\
\hline $15 \%$ & $\begin{array}{l}\text { Comunicação entre os softwares não é 100\% } \\
\text { automatizada }\end{array}$ & $\begin{array}{c}\text { (MATTHEWS et al.,2015; CHEN et al., 2013; KONIG et } \\
\text { al., 2012; LIU; AL-HUSSEIN; LU, 2015) }\end{array}$ \\
\hline $11 \%$ & $\begin{array}{c}\text { Desconsidera tarefas como escavações, limpeza de } \\
\text { terreno e fatores de risco externo }\end{array}$ & $\begin{array}{l}\text { (WANG et al., 2014; KIM et al., 2013; LIU; AL-HUSSEIN; } \\
\text { LU, 2015) }\end{array}$ \\
\hline $7 \%$ & Custo alto de implementação e treinamento & (PITAKE; PATIL, 2013; WANG; CHIEN, 2014); \\
\hline $7 \%$ & $\begin{array}{c}\text { Visualização ineficiente para atividades internas e } \\
\text { externas simultâneas }\end{array}$ & $\begin{array}{l}\text { (BRITO; FERREIRA, 2015; BIOTTO; FORMOSO; ISATTO, } \\
\text { 2015); }\end{array}$ \\
\hline $7 \%$ & $\begin{array}{c}\text { Depende do bom funcionamento de Hardware e da } \\
\text { expertise do gestor para a estimativa de tempo de } \\
\text { duração de atividades }\end{array}$ & (TSERNG; HO; JAN, 2014; LIU; AL-HUSSEIN; LU, 2015) \\
\hline $4 \%$ & $\begin{array}{l}\text { Falta de padronização da dados e documentos entre as } \\
\text { equipes de projeto }\end{array}$ & (MATTHEWS et al., 2015); \\
\hline
\end{tabular}

Fonte: Os autores.

Quadro 4-Síntese dos benefícios identificados e referência dos autores que os mencionaram

\begin{tabular}{|c|c|c|}
\hline Recorrência & BENEFÍCIOS & REFERÊNCIA \\
\hline $44 \%$ & Otimização do processo construtivo & $\begin{array}{l}\text { (SAAD et al. 2015; HU; ZHANG 2010; LIU; AL-HUSSEIN; } \\
\text { LU, 2015; LI et al., 2009, FENG; CHEN; HUANG, 2010; } \\
\text { CHEN et al., 2013; BIOTTO; FORMOSO; ISATTO, 2013; } \\
\text { LUKE et al., 2014; MATTHEWS et al., 2015; LIU et al, } \\
\text { 2014; BRITO; FERREIRA 2015; BIOTTO; FORMOSO; } \\
\text { ISATTO, 2012; AHANKOOB et al., 2012; MENDES et al., } \\
\text { 2014) }\end{array}$ \\
\hline $34 \%$ & Melhoria da comunicação através da integração & $\begin{array}{l}\text { (BRITO; FERREIRA, 2015; BIOTTO; FORMOSO; ISATTO, } \\
\text { 2015; MENDES et al., 2014; PITAKE; PATIL, 2013; LIU; } \\
\text { AL-HUSSEIN; LU, 2015; WU; HSIEH, 2012; HU; ZHANG, } \\
\text { 2010; SAAD et al., 2015; CHEN et al., 2013; TSERNG; } \\
\text { HO; JAN, 2014; ROLFSEN; MERSCHBROCK, 2016) }\end{array}$ \\
\hline
\end{tabular}


SILVA, Paula Heloisa da; CRIPPA, Julianna; SCHEER, Sergio.

BIM 4D no planejamento de obras: detalhamento, benefícios e dificuldades

Quadro 4-Síntese dos benefícios identificados e referência dos autores que os mencionaram

\begin{tabular}{|c|c|c|}
\hline Rerorrôncia & RENEFÍCIOS & \\
\hline $28 \%$ & Redução do retrabalho & $\begin{array}{l}\text { (MENDES et al., 2014; SAINI; MHASKE, 2013; PITAKE; } \\
\text { PATIL, 2013; FENG; CHEN; HUANG, 2010; LI et } \\
\text { al.,2009; MOON, H. et al.,2013; SAAD et al., 2015; } \\
\text { WANG; LEITE, 2012; MATTHEWS et al., 2015) }\end{array}$ \\
\hline $25 \%$ & Redução do tempo de obras & $\begin{array}{c}\text { (MENDES et al., 2014; SAINI; MHASKE, 2013; PITAKE; } \\
\text { PATIL, 2013; FENG; CHEN; HUANG, 2010; LI et } \\
\text { al.,2009; MOON, H. et al.,2013; SAAD et al., 2015; } \\
\text { WANG; LEITE, 2012) }\end{array}$ \\
\hline $25 \%$ & $\begin{array}{l}\text { Análises e alocação de recursos mais eficientes } \\
\text { associado à estimativa de produtividade }\end{array}$ & $\begin{array}{c}\text { (WANG et al., 2014; KIM et al., 2013; BIOTTO; } \\
\text { FORMOSO; ISATTO, 2012; LIU et al, 2014; LI et al., } \\
\text { 2009; MOON et al., 2013; SAAD et al., 2015; WANG; } \\
\text { LEITE, 2012) }\end{array}$ \\
\hline $22 \%$ & Simulações do processo construtivo & $\begin{array}{l}\text { (WANG; LEITE, 2012, WANG et al., 2014, KIM et al., } \\
\text { 2013, MENDES JUNIOR et al., 2014, LUKE et al., 2014, } \\
\text { BIOTTO; FORMOSO; ISATTO, 2015; ZHANG; LI, 2010.) }\end{array}$ \\
\hline $19 \%$ & Otimização do cronograma & $\begin{array}{l}\text { (WANG et al.,2014; BRITO; FERREIRA, 2015; CHEN et } \\
\text { al., 2013; Ll et al., 2009; LIU; AL-HUSSEIN; LU, 2015; } \\
\text { SAAD et al., 2015) }\end{array}$ \\
\hline $19 \%$ & $\begin{array}{l}\text { Melhoria na visualização e interpretação do cronograma } \\
\qquad \text { de obras }\end{array}$ & $\begin{array}{l}\text { (WANG et al.,2014; BRITO; FERREIRA, 2015; CHEN et } \\
\text { al., 2013; LI et al., 2009; LIU; AL-HUSSEIN; LU, 2015; } \\
\text { SAAD et al., 2015) }\end{array}$ \\
\hline $16 \%$ & Integração e automação do sistema de monitoramento & $\begin{array}{l}\text { (TSERNG; HO; JAN, 2014; MATTHEWS et al., 2015; } \\
\text { CHEN et al., 2013; FENG; CHEN; HUANG, 2010; WANG; } \\
\text { LEITE, 2012) }\end{array}$ \\
\hline $16 \%$ & Controle virtual de obras & $\begin{array}{c}\text { (TSERNG; HO; JAN, 2014; MATTHEWS et al., 2015; } \\
\text { CHEN et al., 2013; FENG; CHEN; HUANG, 2010; WANG; } \\
\text { LEITE, 2012) }\end{array}$ \\
\hline $16 \%$ & $\begin{array}{l}\text { Melhoria do arranjo físico e logístico do canteiro de } \\
\text { obras }\end{array}$ & $\begin{array}{l}\text { (AHANKOOB et al., 2012; BIOTTO; FORMOSO; ISATTO, } \\
\text { 2012; BRITO; FERREIRA, 2015; WANG; LEITE, 2012; } \\
\text { PITAKE; PATIL, 2013) }\end{array}$ \\
\hline $16 \%$ & Detecção de incompatibilidades & $\begin{array}{c}\text { (AHANKOOB et al., 2012; MENDES JUNIOR et al., } \\
\text { 2014; LUKE et al., 2014; HU; ZHANG, 2010; SAAD et al. } \\
\text { 2015) }\end{array}$ \\
\hline $16 \%$ & Extração de quantitativos automática & $\begin{array}{c}\text { (KIM et al., 2013; AHANKOOB et al., 2012; LIU et al., } \\
\text { 2014; FENG; CHEN; HUANG, 2010; WANG; LEITE, } \\
\text { 2012) }\end{array}$ \\
\hline $13 \%$ & Criação de base de dados detalha e consistente & $\begin{array}{c}\text { (CHEN et al., 2013; SAAD et al., 2015; KONIG et al., } \\
\text { 2012; PARK; CAI, 2017) }\end{array}$ \\
\hline $6 \%$ & Coordenação em 3D & (PITAKE; PATIL, 2013; AHANKOOB et al., 2012) \\
\hline $6 \%$ & Auxilia na identificação de recursos sobrecarregados & (MOON et al., 2013; LIU et al., 2014) \\
\hline
\end{tabular}

Fonte: Os autores.

\section{Conclusão}

Com base nos dados apresentados no Quadro 4, foi possível verificar que majoritariamente as dificuldades estão relacionadas à implementação da tecnologia. Dessa forma, deduz-se que alguns dos prováveis fatores que acarretam neste problema devem ser: a falta de treinamento especializado para a implementação da nova metodologia e/ou, até mesmo, a falha na mudança de mentalidade dos envolvidos que precisam entender e aceitar o processo integrado como um todo. A segunda maior dificuldade está no processo em si que é trabalhoso, envolvendo intensa atualização do modelo e associação com o cronograma.

A relação de benefícios e dificuldades provenientes da RSL, possibilitou concluir que a viabilidade da implementação de BIM 4D é diretamente dependente de algumas decisões que devem ser tomadas no Startup do Projeto como: utilização unânime de ferramentas BIM, padronizações de documentos e processos de modelagem, definição de fluxos de informação, entre outros. Afinal, como posto por Ahankoob et al. (2012), 
grande parte dos problemas associados à aplicação da metodologia BIM, pode ser resolvido em contrato por meio da Entrega Integrada de Projeto (Integrated Project Delivery- IPD), onde se estabelece a padronização de documentos, softwares, formatação de arquivos, entre outros, para viabilizar a integração no processo projetual.

Quanto aos benefícios, a simulação dinâmica da construção e a integração da comunicação proveniente da tecnologia BIM 4D, resultam na otimização do processo construtivo suscitando a eficiência do PCO por meio da disponibilização de informações consistentes (ZHANG; LI, 2010, MOON et al., 2013, PARK; CAI, 2015). Um exemplo, é a redução de $7,6 \%$ no tempo destinado a execução de obra, em virtude do uso da Modelagem 4D, evidenciado por Chen et al. (2013).

Por fim, a presente RSL permite concluir que o uso de BIM 4D para o PCO, apesar de ainda apresentar algumas dificuldades, se evidencia viável. A modelagem BIM 4D auxilia no provimento de diretrizes de otimização do processo tradicional de $\mathrm{PCO}$, trazendo soluções para grande parte dos problemas inerentes ao método comum. Além disso, contribui significativamente para a redução dos trabalhos manuais por meio da interoperabilidade e integração de sistemas de comunicação.

O BIM 4D ainda possui grande potencial de desenvolvimento, devido às dificuldades enfrentadas para a implementação efetiva da tecnologia. De forma a contribuir para esse desenvolvimento, sugerimos: a realização de uma survey a fim de validar, ou não, os benefícios e dificuldades enfrentados pelo uso de BIM 4D, perante a realidade do mercado nacional ou regional; realização de estudos empíricos ou de caso para verificar a fonte das dificuldades do uso de BIM 4D para PCO; realização de survey nas instituições de ensino e treinamento acerca da maturidade do conhecimento obtido sobre a tecnologia BIM.

\section{Referências}

AHANKOOB, Alireza et al. Optimizing Construction Scheduling Through Use of Building Information Modeling in Construction Industry. In: MANAGEMENT IN CONSTRUCTION RESEARCH ASSOCIATION, 2012, Malásia. Proceedings [...]. Malásia: Universiti Teknologi Malaysia, 2012. p. 166-171.

BIOLCHINI, J.C.D.A.; MIAN, P.G.M.; CRUZ, A.C. NATALI; CONTE, T.U.; TRAVASSOS, G.H. Scientific research ontology to support systematic review in software engineering. Advanced Engineering Informatics. v.21, p. 133-151.2007. DOI: https://doi.org/10.1016/j.aei.2006.11.006.

BIOTTO, C. N.; FORMOSO, C. T.; ISATTO, E. L. Método Para o Uso da Modelagem BIM 4D na Gestão da Produção em Empreendimentos de Construção. In: SIMPÓSIO BRASILEIRO DE QUALIDADE DO PROJETO NO AMBIENTE CONSTRUÍDO, 3., 2013, Campinas. Anais [... ]. Campinas: UNICAMP, 2013. p. 882-893.

BIOTTO, C. N.; FORMOSO, C. T.; ISATTO, E. L. O Uso da Modelagem BIM 4D na Projeto e Gestão de Sistemas de Produção em Empreendimentos de Construção. In: ENCONTRO NACIONAL DE TECNOLOGIA DO AMBIENTE CONSTRUÍDO, 14., Juiz de Fora, 2012, Juiz de Fora. Anais [...]. Juiz de Fora: ANTAC, 2012.

BIOTTO, C.; FORMOSO, C. T.; ISATTO, E. L.. Uso de modelagem 4D e Building Information Modeling na gestão de sistemas de produção em empreendimentos de construção. Ambiente Construído v. 15, n. 2, p. 79-96, jun. 2015. DOI: http://dx.doi.org/10.1590/s1678-86212015000200015.

BRERETON, O. P. et al. Lessons from Applying the Systematic Literature Review Process within the Software Engineering Domain. The Journal of Systems and Software, v. 80, n. 4, p. 571-583, 2007.

DOI: https://doi.org/10.1016/j.jss.2006.07.009. 
BRITO, D. M. de; FERREIRA, E. de A. M. Avaliação de estratégias para representação e análise do planejamento e controle de obras utilizando modelos BIM 4D. Ambiente Construído, Porto Alegre, v. 15, n. 4, p. $203-223$, out./dez. 2015. DOI: http://dx.doi.org/10.1590/s1678-86212015000400047.

CBIC - CÂMARA BRASILEIRA DA INDÚSTRIA DA CONSTRUÇÃO. Coletânea implementação do BIM para construtoras e incorporadoras: volume 5 formas de contratação BIM. Brasília: CBIC, 2016.

CHEN, S. et al. A framework for an automated and integrated project scheduling and management system. Automation in Construction, v. 35, p. 89-110, 2013. DOI: https://doi.org/10.1016/j.autcon.2013.04.002.

CIC - COMPUTER INTEGRATED CONSTRUCTION Research Program. BIM Project Execution Planning Guide. Version 2.1, The Pennsylvania State University, University Park, PA, USA. 2011. Disponível em: <http://bim.psu.edu>. Acesso em: 12 out. 2015

DRESCH, A.; LACERDA, D. P.; ANTUNES JÚNIOR, J. A. V. Design Science Research: Método de Pesquisa para Avanço da Ciência e Tecnologia. Bookman Editora, 2015.

EASTMAN, C. et al. Strategies for Realizing the Benefits of 3D Integrated Modeling of Buildings for the AEC Industry. In: INTERNATIONAL SYMPOSIUM ON AUTOMATION AND ROBOTICS IN CONSTRUCTION, 19., Washington DC, 2002. Anais [...]. Washington: NIST, 2002.

EASTMAN, C. et al. BIM Handbook: A Guide to Building Information Modeling for Owners, Managers, Designers, Engineers, and Contractors. 2 ed., New Jersey: John Wiley \& Sons, 2011.

FENG, C.; CHEN, Y.; HUANG, J. Using the MD CAD model to develop the time-cost integrated schedule for construction projects. Automation in Construction, v. 19, n. 3, p. 347-356, 2010. DOI:

https://doi.org/10.1016/j.autcon.2009.12.009.

HARTMANN, T. et al. Aligning building information model tools and construction management methods. Automation in construction, v. 22, p. 605-613, 2012. DOI: https://doi.org/10.1016/j.autcon.2011.12.011.

HU, Z.; ZHANG, J. BIM-and 4D-based integrated solution of analysis and management for conflicts and structural safety problems during construction: 2. Development and site trials. Automation in Construction, v. 20, n. 2, p. 167180, 2011. DOI: https://doi.org/10.1016/j.autcon.2010.09.014.

$\mathrm{KIM}$, H. et al. Generating construction schedules through automatic data extraction using open BIM (building information modeling) technology. Automation in Construction, v. 35, p. 285-295, 2013. DOI: https://doi.org/10.1016/j.autcon.2013.05.020.

KITCHENHAM, B. et al. Systematic Literature Reviews in Software Engineering: a systematic literature review. Information and Software Technology, v. 51, n. 1, p. 7-15, 2009. DOI: https://doi.org/10.1016/j.infsof.2008.09.009.

KONIG, M. et al. Intelligent BIM-based construction scheduling using discrete event simulation. IEEE, 2012, p.1-12. DOI: https://doi.org/10.1109/WSC.2012.6465232.

LAISERIN, J. Comparing Pommes and Naranjas. The Laiserin Letter, December 16, Issue 15, 2002.

$\mathrm{LI}$, H.et al. Optimizing construction planning schedules by virtual prototyping enabled resource analysis. Automation in construction, v. 18, n. 7, p. 912-918, 2009. DOI: https://doi.org/10.1016/j.autcon.2009.04.002.

LIU, H.; LEI, Z; LI, H.; AL-HUSSEIN, M. An automatic scheduling approach: Building information modeling-based onsite scheduling for panelized construction. In: CONSTRUCTION RESEARCH CONGRESS, 2014, Atlanta, GA, USA. Proceedings [... ]. Atlanta, mai 19-21, pp. 1666-1675.

LIU, H.; AL-HUSSEIN, M.; LU, M. BIM-based integrated approach for detailed construction scheduling under resource constraints. Automation in Construction, v. 53, p. 29-43, 2015. DOI:

https://doi.org/10.1016/j.autcon.2015.03.008. 
LUKE, W. G.et al. Uso de ferramentas BIM para o melhor planejamento de obras da Construção Civil. In: CONGRESSO BRASILEIRO DE ENGENHARIA DE PRODUÇÃO, 4., 2014, Ponta Grossa. Anais [...]. Ponta Grossa, $03-05$ dez 2014.

MATTHEWS, J. et al. Real time progress management: Re-engineering processes for cloud-based BIM in construction. Automation in Construction, v. 58, p. 38-47, 2015. DOI: https://doi.org/10.1016/j.autcon.2015.07.004.

MENDES JUNIOR, R. et al. Integração da modelagem da informação da construção (BIM) com o planejamento e controle da produção. In: ENCONTRO NACIONAL DE TECNOLOGIA DO AMBIENTE CONSTRUÍDO, 15., 2014, Maceió. Anais [...]. Maceió, 12-14 nov 2014.

MIKULAKOVA, E. et al. Knowledge-based schedule generation and evaluation. Advanced Engineering Informatics, v. 24, n. 4, p. 389-403, 2010. DOI: https://doi.org/10.1016/j.aei.2010.06.010.

MOON, H.et al. BIM-based construction scheduling method using optimization theory for reducing activity overlaps. Journal of Computing in Civil Engineering, v. 29, n. 3, p. 04014048 , 2013. DOI:

https://doi.org/10.1061/\%28ASCE\%29CP.1943-5487.0000342.

PARK, J.; CAI, H. Automatic Construction Schedule Generation Method through BIM Model. Creation.Computing in Civil Engineering, 2015. DOI: https://doi.org/10.1061/9780784479247.077.

PARK, J.; CAI, H. WBS-based dynamic multi-dimensional BIM database for total construction as-built documentation. Automation in Construction v. 77, p. 15-23, 2017. DOI: https://doi.org/10.1016/j.autcon.2017.01.021.

PITAKE, S. A.; PATIL, D. S. Visualization of construction progress by 4D modeling application. International Journal of Engineering Trends and Technology, v. 4, n. 7, 2013.

QUIRK, V. A Brief History of BIM. Archdaily. Disponível em: <http://www.archdaily.com/302490/a-brief-history-ofbim>. Acesso em: 29 abr. 2016.

ROLFSEN, C. N.; MERSCHBROCK, C. Acceptance of Construction Scheduling Visualizations: Bar-charts, Flowlinecharts, Or Perhaps BIM? Procedia Engineering Selected papers from Creative Construction Conference 2016, v. 164, p. 558-566 , 2016. DOI: https://doi.org/10.1016/j.proeng.2016.11.658.

SAAD, M.et al. A Suggested Solution to Improve the Traditional Construction Planning Approach. Jordan Journal of Civil Engineering, v. 9, n. 2, 2015.

SAINI, V. K.; MHASKE, S. BIM based Project Scheduling and Progress Monitoring in AEC Industry. International Journal of Scientific Engineering and Research (IJSER), 2013.

SHEN, W. et al. Systems integration and collaboration in architecture, engineering, construction, and facilities management: A review. Advanced Engineering Informatics, v. 24, n. 2, p. 196-207, 2010. DOI:

https://doi.org/10.1016/j.aei.2009.09.001.

SILVA, P. H. da; SCHEER, S; ARRUDA, R. F. Benefícios, restrições e dificuldades do uso de BIM 4D E 5D para PCO uma RSL. In: SIMPÓSIO BRASILEIRO DE QUALIDADE DO PROJETO NO AMBIENTE CONSTRUÍDO, 5., 2017, João Pessoa. Anais [...]. João Pessoa: ANTAC, 2017.

TSERNG, H.; HO, S.; JAN, S. Developing BIM-assisted as-built schedule management system for general contractors. Journal of Civil Engineering and Management, v. 20, n. 1, p. 47-58, 2014. DOI: https://doi.org/10.3846/13923730.2013.851112.

VA - U.S. DEPARTMENT OF VETERANS AFFAIRS. The VA BIM Guide. v. 1.0, Washington: VA, 2010.

WANG, C. C.; CHIEN, O. The Use of BIM in Project Planning and Scheduling in the Australian Construction Industry. In: INTERNATIONAL CONFERENCE ON CONSTRUCTION AND REAL ESTATE MANAGEMENT, 2014, Kunming, China. Proceedings [... ]. Kunming, China: ASCE, 2014. p. 126-133. 
WANG, L; LEITE, F. Towards Process-aware Building Information Modeling for Dynamic Design and Management of Construction Processes. In: ANNUAL WORKSHOP OF THE EUROPEAN GROUP FOR INTELLIGENT COMPUTING IN ENGINEERING (EG-ICE), 19., 2012, Germany. Anais [... ]. Herrsching, Germany: Technische Universität München, 2012.

WANG, W. et al. Integrating building information models with construction process simulations for project scheduling support. Automation in construction, v. 37, p. 68-80, 2014. DOI:

https://doi.org/10.1016/j.autcon.2013.10.009.

WU, I.; HSIEH, S. A framework for facilitating multi-dimensional information integration, management and visualization in engineering projects. Automation in Construction, v. 23, p. 71-86, 2012. DOI: https://doi.org/10.1016/j.autcon.2011.12.010.

ZHANG, J.; LI, D. Research on 4D virtual construction and dynamic management system based on BIM. In: INTERNATIONAL CONFERENCE ON COMPUTING IN CIVIL AND BUILDING ENGINEERING, 2010, Nottingham, Reino Unido. Proceeding [...]. Reino Unido: The University of Nottingham, 2010.

\section{${ }^{1}$ Paula Heloisa da Silva}

Mestranda no Programa de Pós-graduação em Engenharia da Construção Civil, UFPR. Endereço postal: Rua Tem, Tito Teixeira de Castro, 725, Curitiba, PR, Brasil, 81650-270.

\section{Julianna Crippa}

Mestranda no Programa de Pós-graduação em Engenharia da Construção Civil, UFPR. Endereço postal: Avenida Visconde de Guarapuava, Número 3945, Curitiba, PR, Brasil, 80250-220

\section{Sergio Scheer}

Pesquisador Sênior. Endereço postal: Av. Francisco H dos Santos, 210, Curitiba, PR, Brasil, 81531-970 